\title{
A camera-based scoring system for evaluating performance accuracy during a golf putting task
}

\author{
DaVid L. Neumann and Patrick R. Thomas \\ Griffith University, Southport, Queensland, Australia \\ and Queensland Academy of Sport, Nathan, Queensland, Australia
}

\begin{abstract}
Golf putting accuracy is often evaluated by measuring the distance that the ball finishes from the hole. However, accuracy is a function of line and length, and distance-from-hole measures confound these two factors. A scoring system for evaluating putting accuracy is described that enables the efficient measurement of errors in line and length. A camera placed above the hole takes digital photographs of the final position of the ball. A custom-developed program written in the National Instruments LabVIEW graphical programming language derives a variety of accuracy measures from these photographs, including distance from the hole, angle of error, distance short or long from the hole, and distance left or right from the hole. Evaluation of the system indicated that the measures were as accurate as manual measurements and were reliable when rescored on separate occasions. The camera-based scoring system presents a number of advantages in the evaluation of putting accuracy and may be extended to examine performance in other sports. The ScorePutting program may be downloaded from the Psychonomic Society's Archive of Norms, Stimuli, and Data, www.psychonomic.org/archive.
\end{abstract}

Performance in sports that require an accurate aim, such as archery and rifle shooting, can be evaluated by measuring how far the shot finishes from the target. However, for sports such as basketball, tennis, and football, targets can be missed because of errors in the aim or errors in the force of the shot. In a similar vein, Pelz (2002) identified two requirements for good putting in golf: the accuracy of the initial line (aim) and the precision of rolling the ball at the proper speed. The combination of these two factors determines whether the ball finishes in the hole. He argued that aim, also referred to as direction, is a critical aspect of putting, with most golfers substantially underestimating the true break of a putt. The golfer must also anticipate how the speed and contours of the putting surface relate to the force that needs to be imparted on the ball (Fairweather, Button, $\&$ Rae, 2002). Performance measures should, therefore, be sensitive to both of these variables.

The most commonly used measure in the evaluation of golf stroke accuracy is the distance the ball finishes from the target (e.g., Beilock \& Carr, 2001; Beilock, Carr, MacMahon, \& Starkes, 2002; Crews \& Landers, 1993). An alternative method is to draw concentric circles of different radii around the target and award increasingly higher points to balls finishing in zones closer to the target (e.g., Guadagnoli \& Holcomb, 1999; Wulf, Lauterbach, \& Toole, 1999). However, neither of these approaches shows whether the ball missed the target because of an error in direction and/or an error in the force of the shot. Such combined measures may be less sensitive to the influence of psychological factors (e.g., anxiety, attentional focus strategy) on the different aspects of performance if these factors influence one aspect of performance and not another. A scoring system that is capable of deriving performance measures sensitive to line and length thus has the potential to advance the methods currently used in sport psychology research.

The present report describes the development and evaluation of a new method for measuring putting performance. The method comprises four steps. First, an apparatus that can take digital photographs of the area around the target (e.g., the hole) is set up. Second, calibration of the system to enable the performance measures to be translated into real measurement units (e.g., centimeters, degrees of error) is completed. Third, photographs are taken of the final position of the ball during the testing of a research participant. Fourth, the digital photographs are scored with the use of computer software to derive a range of performance measures based on the line and length of the putt.

\section{Apparatus Used to Obtain \\ the Digital Photographs}

The apparatus used to obtain the digital photographs is shown in Figure 1. It consisted of a digital camera that was attached to a tripod and boom. The digital camera was a Sony Mini DV Handycam (Model DCRHC42) fitted with a 25-mm 0.6× Sony Wide Conversion Lens (Model VCL0625S). The camera can take moving videos or still digital photographs, although only the still photographic function was used in this application. It has a number of configurable settings, and of these, the highest resolution $(1,152 \times 864$

D. L. Neumann, d.neumann@griffith.edu.au 


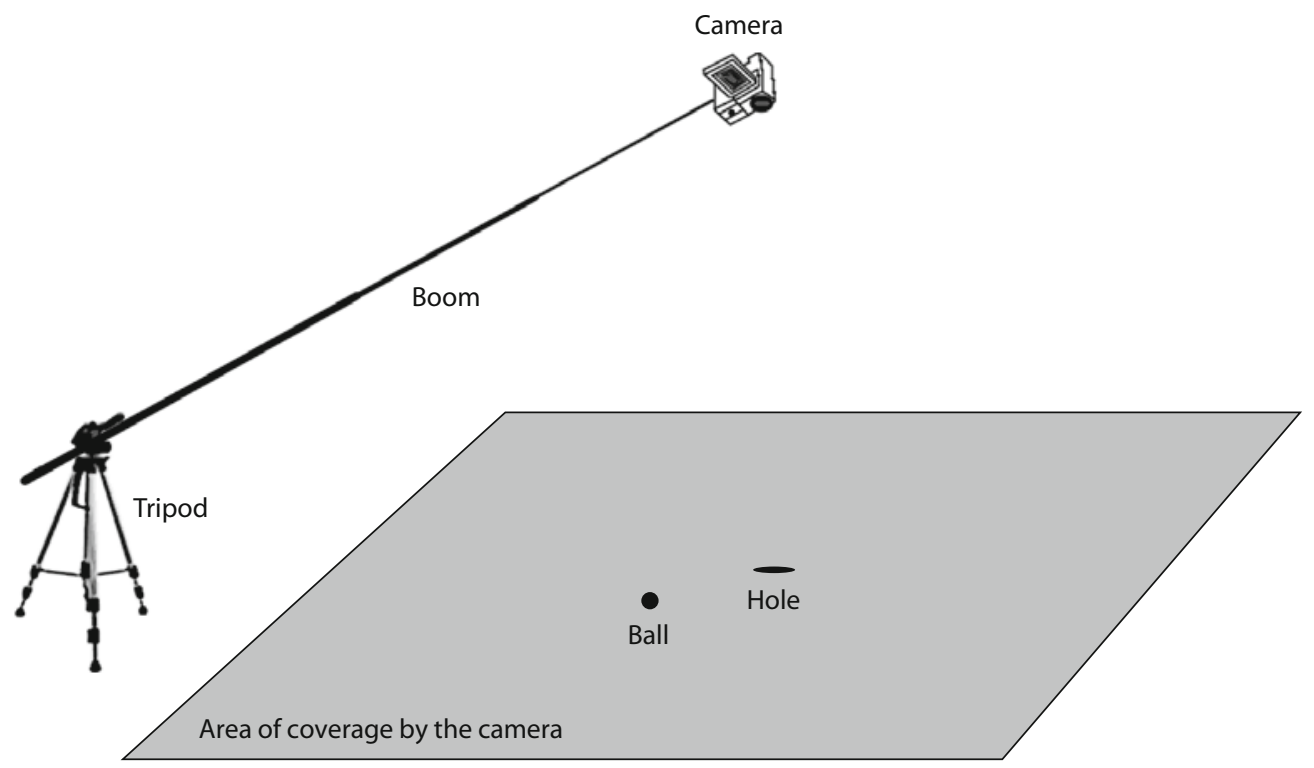

Figure 1. A schematic of the setup for the apparatus used to take photographs of the area around the target hole.

pixels), the widest lens aperture (i.e., no zoom), and the date/ time function were employed. The camera runs on its own battery power, enabling the system to be used in locations without an accessible power supply. The wide conversion lens was attached to the camera to ensure that a sufficiently large area around the target could be photographed.

The camera was attached to a tripod and boom. The tripod was a standard metal one used for photography and was $1.42 \mathrm{~m}$ in height when erect. Attached to the tripod was a telescopic carbon fiber boom pole that could be extended from 0.90 to $3.9 \mathrm{~m}$. The camera was attached to the end of the boom, and due to its weight (470 g, including battery), it was necessary to stabilize the tripod with small shot bags, to avoid having the apparatus tip over. The tripod and boom allowed the camera to be suspended $3.4 \mathrm{~m}$ above the target hole. Suspending the camera in excess of this height would allow the photographs to capture a wider area around the target hole. However, we found that the resulting area coverage of approximately $5 \times 3.75 \mathrm{~m}$ was adequate to capture all but the most erroneous putts when novice, experienced, and elite athletes made a series of 20 putts $2.4 \mathrm{~m}$ from the hole.

\section{System Calibration}

It is necessary to calibrate the system prior to recording a participant's performance. The first step in the calibration is to ensure that the camera lens is suspended parallel to the level ground around the target hole. A circular bubble level attached to the camera allowed this to be done. The second step is to take a photograph of calibration objects to provide references for later scoring. The two calibration objects are highlighted in the photograph in Figure 2. One of these objects is a 1-m bar that can be placed at any location in the photograph. The second object is a rope that is pulled straight along a line running from the center of the target hole to the origin of the putt. In most cases, the putt origin will lie outside the photographed area. The calibration objects provide a means to measure distances and angle of error in real units.

\section{Taking Photographs During Testing of a Research Participant}

The testing of research participants is efficient and proceeds quickly. All that is required is that a photograph of the final position of the ball is taken after each putt. The camera is supplied with a remote control to allow the photographs to be taken even though the camera is suspended. When the appropriate button on the remote control is pressed, the camera emits an audible sound to provide verification that a photograph has been taken. After the photographs have been taken, the ball can be removed from the green, and the participant can proceed with the next attempt. All photographs are stored on a memory stick in the camera in .jpg format. The photographs can be saved with an arbitrary name (e.g., DSC04561.JPG) that increases in count with each successive photograph. The use of the date and time setting on the camera provides a second means by which the data can be tracked back to a particular participant.

\section{Computer Software Used for Scoring}

Putting performance was scored through the use of a custom-developed computer program written within the National Instruments Laboratory Virtual Instrumentation Engineering Workbench (LabVIEW) programming environment. LabVIEW provides a graphical interface to develop custom-written programs that can be ported to a variety of platforms, including Windows, Mac OS, and Linux. This programming environment has been used to develop other data scoring and analysis programs in 


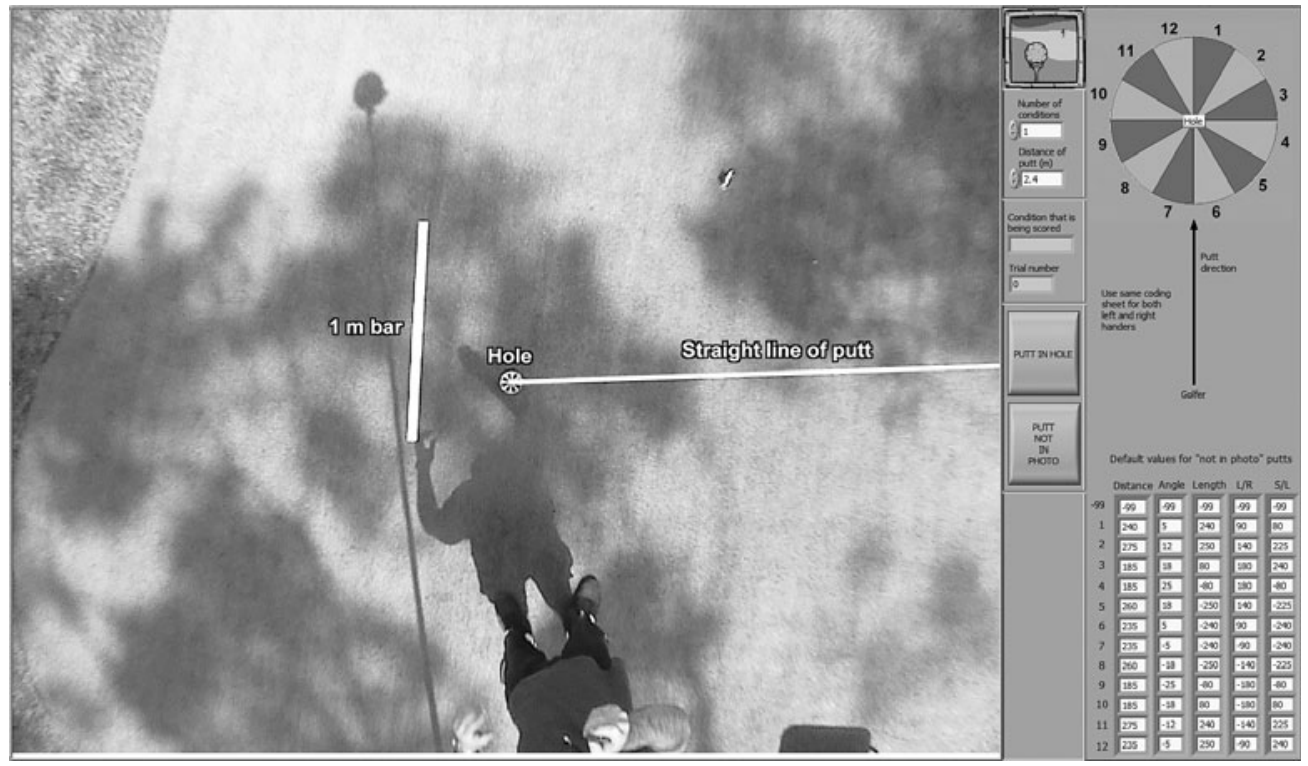

Figure 2. The graphic user interface for the ScorePutting program written in the National Instruments LabVIEW programming language. The majority of the interface shows the photograph of the area around the target hole. The photograph in this figure shows the calibration objects of the 1-m bar and the rope. The interface also contains a number of controls and indicators in the right panel that are used during scoring.

behavior research (e.g., Angrilli, 1995; Duley, Janelle, \& Coombes, 2004). Programming within LabVIEW involves interacting with a front panel that provides a means to develop the graphic user interface (GUI) and a diagram panel that provides a means to set the parameters and flow of data through the program. Programs written in LabVIEW are termed virtual instruments, or VIs. The custom-developed VI used to evaluate putting accuracy in this project was called ScorePutting and contained a number of sub-VIs that used algorithms for calculating the various performance parameters.

The GUI for ScorePutting is shown in Figure 2. The majority of the screen is dedicated to displaying the photographs. The right panel of the screen contains several controls and indicators to assist with scoring. Two controls that must be set before starting the program are Number of conditions and Distance of putt $(\mathrm{m})$. By specifying the number of conditions, the user can employ the same calibration values and saved data file to score multiple experimental conditions from the same participant. This might occur, for example, if the participant used different performance routines in different blocks of putts. The distance of the putt needs to be specified because the origin of the putt will rarely be shown in the photograph. The final parameters that can be set by the user are the Default values for "not in photo" putts. This is to be used for those rare occasions on which a putt has been particularly poor, so that it does not lie within the photographed area. In these cases, the experimenter should code the putt according to the direction in which the putt finished, using the coding sheet shown in the top right-hand corner of the GUI. This will allow estimated values or missing values, according to the user's preference, to be used. The estimated values can be calculated manually for the 12 different coded locations. The exact values will vary according to the individual setup, because it will depend on the coverage of the photographed area.

The sequence of operations that occurs upon starting ScorePutting is shown in Figure 3. The program initially prompts for the filename to which to save the data. Identifying characteristics of the participant (name/code and handedness) are requested and saved in the data file. The latter characteristic may also be used to determine whether shots are missed by a push/slice or pull/hook, if desired. The .jpg file for the calibration photograph is displayed next. The calibration proceeds by (1) clicking on the end points of the $1-\mathrm{m}$ calibration bar placed in the photo, (2) clicking on two points along the straight line that runs from the center of the hole to the origin of the putt, and (3) clicking on the center of the hole. A dot appears on the photograph for visual verification during this process, and there is an option to repeat the calibration if an error is made. After the calibration values have been accepted, the performance data may be scored. All that is required is to browse to the folder that contains the .jpg photographs for the condition to be scored and select the folder. The program will automatically load each photograph sequentially in alphanumerical order for scoring. If more than one condition has been specified to be scored, the user will be prompted for the folder that contains the photographs for the next condition, and the procedure repeats.

The scoring of the photographs proceeds quickly after calibration. If the ball finished in the hole, the user clicks on the "PUTT IN HOLE" button and values are automatically recorded (e.g., distance from hole $=0$, angle of error $=0$ ). If the ball finished outside of the photographed area, the user clicks on the "PUTT NOT IN PHOTO" but- 


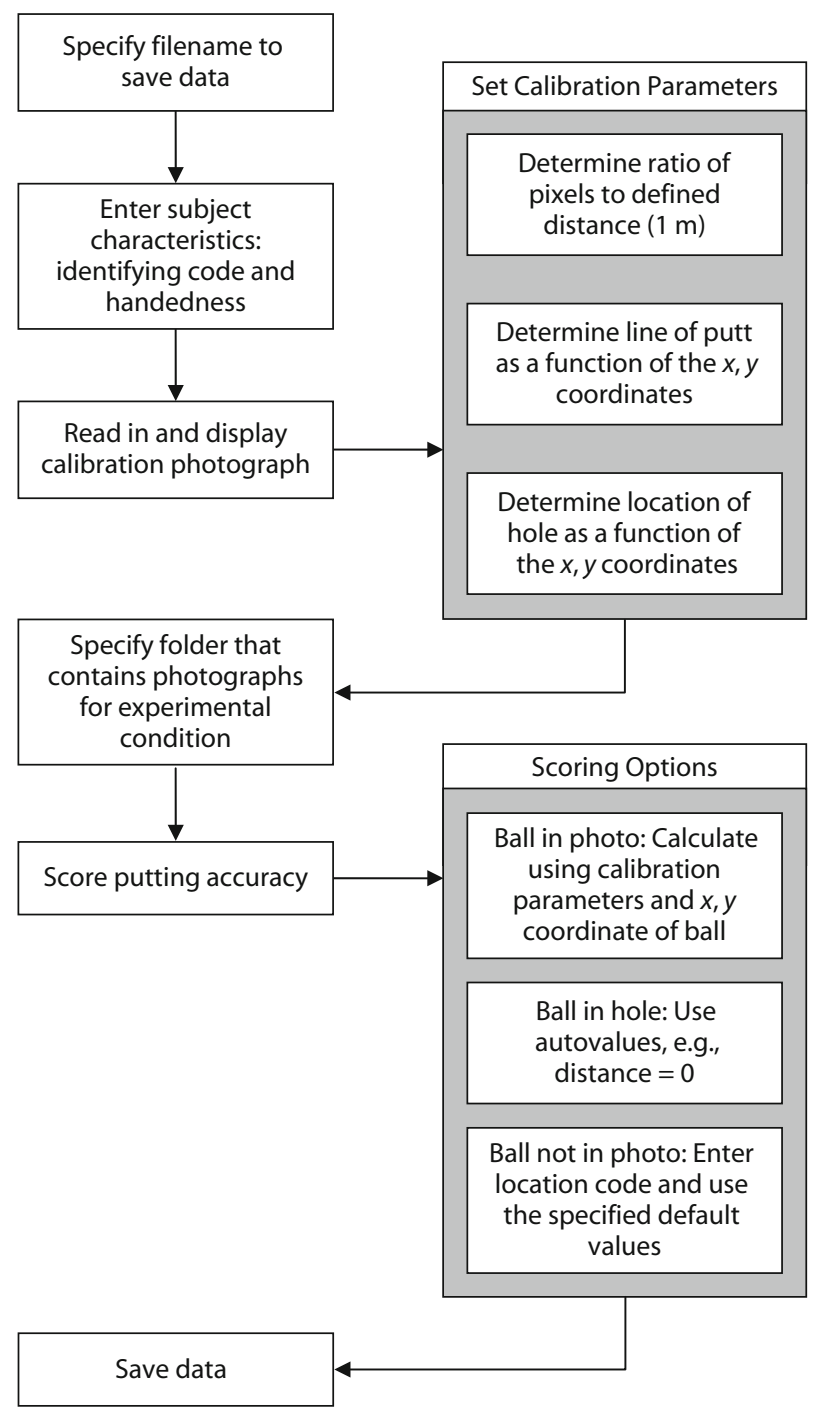

Figure 3. A flowchart showing the sequence of operations that occur to evaluate putting performance with the ScorePutting program.

ton. A dialog box will be displayed, prompting the user to specify the numerical code for the appropriate estimated values. Finally, if the ball finished in the photograph but not in the hole, the user simply clicks on the ball to measure performance. In this case, the program will use a series of algorithms to calculate the various parameters to measure performance. The performance measures that are calculated are shown in Figure 4 and include (a) distance short or long, (b) length error (distance of ball from origin minus distance from origin to hole), (c) angle of error, (d) distance left or right, and (e) distance from hole. All except the angle of error are expressed in centimeters, and measures a, b, and d are expressed as either positive or negative values to indicate the direction of the error (e.g., negative values indicate that the final distance was short or left).

The first two measures ( $a$ and b) reflect accuracy in the force of the putt. In addition to providing a measure of how well a golfer can read the speed of the green, such measures may be sensitive to emotional factors (e.g., anxiety can increase muscular tension and make it difficult to lag a putt effectively). The next two measures (c and d) reflect accuracy in aim, identifying errors in direction as well as any consistent patterns in those errors, such as pushing or pulling putts. Separate evaluations of distance and aiming errors will indicate whether a putt has missed because of either or both of these factors. The final measure, distance from hole, reflects a combination of distance and aim. It can provide a common metric for comparisons with the results in previous studies in which distance and aiming were not separated in evaluating performance (e.g., Beilock \& Carr, 2001; Beilock et al., 2002; Crews \& Landers, 1993).

The calculation of the various performance parameters is based on the principle of dividing the photograph into $x$ and $y$-coordinates. A $1,152 \times 864$ pixel photograph thus consists of 1,152 values along the $x$-axis and 864 values along the $y$-axis, although the axes can be extended indefinitely to allow the position of objects not in the photograph to be specified (e.g., the origin of the putt). The location of the hole and the final position of the ball can thus be specified by separate $x, y$ coordinates, and the line of the putt can be described by the use of a regression equation. Using the various known $x, y$ coordinates and the line of the putt, the methods of trigonometry are applied to calculate the various performance parameters. In the case of the distance measures, the values are originally calculated in pixels and subsequently converted to centimeters. The conversion is possible because the calibration of the system uses the $1-\mathrm{m}$ bar to determine the ratio of how many pixels correspond to $1 \mathrm{~m}$. The final measure reflects the distance from the center of the ball to the center of the relevant reference (e.g., the hole). At the completion of scoring of all the photographs, all the measured parameters are saved within a text-formatted data file.

\section{Evaluation of the System}

To evaluate the accuracy and consistency of the system, we took a sample of 20 photographs in which the ball was placed randomly from 12 to $210 \mathrm{~cm}$ in each of the four quadrants around the hole. Photographs were taken at each ball position, and the distance of the ball from the hole was noted on a tape measure. When the photographs were subsequently scored, the correlation between the computer-calculated distance from the hole and the manually measured distance from the hole was very high $(r=.9996, p<.001)$. Test-retest reliability was also very high when the photograph data were rescored 1 week later, with a correlation between the two sets of scores of $r=$ $.9999(p<.001)$.

\section{Relative Merits of the System}

The camera-based scoring system presents a number of advantages that encourage its use in the evaluation of putting performance. It provides a permanent photographic record of the participant's performance. This can be advantageous if different performance measures need to be calculated from the same study at a later date. The camera-based system considerably simplifies the testing 


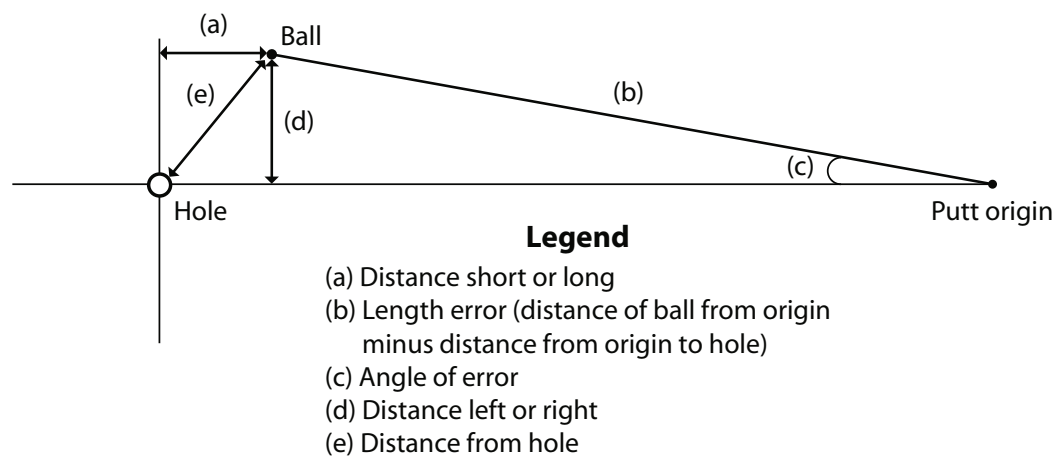

Figure 4. The five performance measures derived from the photographs when the ball did not finish in the hole.

procedure by eliminating the need for the experimenter to make manual measurements. Apart from the significant ergonomic advantages, the time taken to test a participant is substantially reduced. The biggest strength of the system is, however, that it allows several parameters of performance to be evaluated simultaneously. The common measure of distance from the hole is complemented by measures that separate performance in line and length to determine whether differences between groups of participants or experimental conditions influence one or both performance factors. In this way, it may be easier to identify systematic errors that occur during testing (e.g., pulling a ball when putting under pressure).

The camera-based scoring system also has a number of features that place limitations on its use. When used outdoors, it can be influenced by weather conditions, in that strong winds can move the camera and it cannot be used when it is raining. However, it would be unlikely that the system would be used under such conditions, since they may also hinder the participant's performance. The camera, although portable, is best used when all putts are directed at one hole, since it would take some time to move the camera from one hole to another. Finally, the system was developed and evaluated for putts taken on a flat, even surface. Some of the performance measures, such as angle of error, may not be readily interpretable when breaking putts are used.

\section{Conclusions}

The camera-based scoring system that has been developed has shown promise as a means by which putting performance can be evaluated. The use of performance measures that are sensitive to errors in line and length has the potential to enrich the evaluation of a participant's putting ability. There is potential for the general system to be extended to other applications. For instance, the use of a video camera to capture moving pictures may allow additional measures to be captured (e.g., pace) or might allow evaluation of performance during breaking putts. It may also be possible to apply the same principles to evaluate performance during other aiming tasks that require accuracy in line and length, such as basketball shooting. By capturing richer accuracy measures, it may be possible to provide a more fine-grained analysis of those psychological and physical factors that influence performance during training or competition.

\section{AUTHOR NOTE}

This research was supported under Australian Research Council's Linkage Projects Funding Scheme (Project LP0667727). Thanks to Minhtri Pham and Xuesong Le for computer programming assistance and to Martin Hampson for assistance during evaluation and testing. Correspondence concerning this article should be addressed to D. L. Neumann, School of Psychology, Griffith University, Gold Coast Campus, Southport, QLD 4222, Australia (e-mail: d.neumann@griffith.edu.au).

\section{REFERENCES}

ANGRILLI, A. (1995). PSAAL: A LabVIEW 3 program for data acquisition and analysis in psychophysiological experiments. Behavior Research Methods, Instruments, \& Computers, 27, 367-374.

BeILOCK, S. L., \& CARR, T. H. (2001). On the fragility of skilled performance: What governs choking under pressure? Journal of Experimental Psychology: General, 130, 701-725.

Beilock, S. L., Carr, T. H., MacMahon, C., \& Starkes, J. L. (2002). When paying attention becomes counterproductive: Impact of divided versus skill-focused attention on novice and experienced performance of sensorimotor skills. Journal of Experimental Psychology: Applied, 8, 6-16.

Crews, D. J., \& LANDERs, D. M. (1993). Electroencephalographic measures of attentional patterns prior to the golf putt. Medicine \& Science in Sports \& Exercise, 25, 116-126.

Duley, A. R., Janelle, C. M., \& Coombes, S. A. (2004). An opensource LabVIEW application toolkit for phasic heart rate analysis in psychophysiological research. Behavior Research Methods, Instruments, \& Computers, 36, 778-783.

Fairweather, M. M., Button, C., \& Rae, I. (2002). A critical examination of motor control and transfer issues in putting. In E. Thain (Ed.), Science and golf IV: Proceedings of the World Scientific Congress of Golf (pp. 100-112). London: Routledge.

GuAdaGnOLI, M., \& Holcomb, W. (1999). Variable and constant practice: Ideas for successful putting. In M. R. Farrally \& A. J. Cochran (Eds.), Science and golf III: Proceedings of the World Scientific Congress of Golf (pp. 261-270). Champaign, IL: Human Kinetics.

Pelz, D. (2002). Dave Pelz's putting bible. London: Aurum.

Wulf, G., Lauterbach, B., \& Toole, T. (1999). Learning advantages of an external focus of attention in golf. Research Quarterly for Exercise \& Sport, 70, 120-126.

\section{ARCHIVED MATERIALS}

The following materials associated with this article may be accessed through the Psychonomic Society's Norms, Stimuli, and Data archive, www.psychonomic.org/archive. 
To access these files, search for this article using the journal (Behavior Research Methods), the first author's name (Neumann), and the publication year (2008). There are two downloadable archives.

FILE: Scoreputting.zip

DESCRIPTION: This archive contains the LabVIEW VI files required to run the ScorePutting program. You must have LabVIEW version 7.1 or higher installed on the computer to run these files. The main file is called ScorePutting.vi, and the sub-VIs used by it are also included in the archive. It is possible to modify the LabVIEW VI files with the LabVIEW program, if necessary, to customize the ScorePutting program to meet individual needs.

FILE: InstallScorePutting.zip
DESCRIPTION: This archive contains a self-extracting archive of files. The files include an .exe version of the ScorePutting program, the LabVIEW runtime environment, and the application installer for Microsoft Windows targets. Installation of these files will allow the ScorePutting program to run on computers in which the LabVIEW program is not installed. However, it is not possible to modify the LabVIEW VI files that were used to compile the executable file.

AUTHOR’s E-MAIL ADDRESS: d.neumann@griffith.edu.au.

(Manuscript received September 12, 2007; revision accepted for publication February 1, 2008.) 